PROCEEDINGS OF THE

AMERICAN MATHEMATICAL SOCIETY

Volume 139, Number 8, August 2011, Pages 2783-2791

S 0002-9939(2010)10783-9

Article electronically published on December 29, 2010

\title{
POSITIVE ENTROPY HOMEOMORPHISMS OF CHAINABLE CONTINUA AND INDECOMPOSABLE SUBCONTINUA
}

\author{
CHRISTOPHER MOURON \\ (Communicated by Alexander N. Dranishnikov)
}

\begin{abstract}
It is shown that if $X$ is a chainable continuum and $h: X \longrightarrow X$ is a homeomorphism such that the topological entropy of $h$ is greater than 0 , then $X$ must contain an indecomposable subcontinuum. This answers a question of Barge.
\end{abstract}

\section{INTRODUCTION}

In dynamics, topological entropy is a number in $[0, \infty]$ that gives a measure of the rate of local expansion of a function on a space. The connection between entropy and the dynamics of a continuous function is well documented. Many simple functions such as the tent map have positive entropy. However, for a homeomorphism to have positive entropy, it appears that the local structure must be complex. For example, in 4, it is shown that no homeomorphism of a regular continuum can have positive entropy. In [5] and [6], Ye showed that homeomorphisms of hereditarily decomposable chainable continua that are induced by square commuting diagrams on inverse systems of intervals must have zero entropy. In this paper, it is shown that every chainable continuum that admits a positive entropy homeomorphism must contain a nondegenerate indecomposable subcontinuum. Indecomposable continua are known to have a very complicated local structure. This answers a well-known question by Barge [2].

A continuum $X$ is a compact connected metric space. Let d be the metric on $X$ and if $A, B \subset X$, then define $\mathrm{d}(A, B)=\inf \{\mathrm{d}(a, b) \mid a \in A$ and $b \in B\}$. A map is a continuous function. A continuum $X$ is decomposable provided there exist proper subcontinua $H$ and $K$ such that $X=H \cup K$. A continuum is indecomposable if it is not decomposable. If $\mathcal{U}$ is a collection of open sets, the mesh of $\mathcal{U}$ is defined as $\operatorname{mesh}(\mathcal{U})=\sup \{\operatorname{diam}(U): U \in \mathcal{U}\}$. A chain $\mathcal{C}$ is an indexed collection of open sets $\left[C_{1}, C_{2}, \ldots, C_{n}\right]$ such that $C_{i} \cap C_{j} \neq \emptyset$ if and only if $|i-j| \leq 1$. A chain is proper if $\bar{C}_{i} \cap \bar{C}_{j} \neq \emptyset$ if and only if $|i-j| \leq 1$. Here, $C_{1}$ and $C_{n}$ are the endlinks of the chain. A continuum $X$ is chainable if for every $\epsilon>0$ there exists a chain covering $X$ such that $\operatorname{mesh}(\mathcal{C})<\epsilon$. Chainable continua are also called arc-like and snake-like continua.

Let $X$ be a compact metric space, $f: X \longrightarrow X$ be a map, and $\mathcal{U}$ be a finite open cover of $X$. Define $N(\mathcal{U})$ be the number of sets in a finite subcover of $\mathcal{U}$ with

Received by the editors August 6, 2008 and, in revised form, July 20, 2010.

2010 Mathematics Subject Classification. Primary 37B45, 37B40; Secondary 54F15.

Key words and phrases. Entropy, chainable continuum, indecomposable continuum.

(C)2010 American Mathematical Society 2783

Reverts to public domain 28 years from publication 
smallest cardinality. If $\mathcal{U}$ and $\mathcal{V}$ are two open covers of $X$, let

$$
\mathcal{U} \vee \mathcal{V}=\{U \cap V \neq \emptyset \mid U \in \mathcal{U}, V \in \mathcal{V}\}
$$

and $f^{-1}(\mathcal{U})=\left\{f^{-1}(U) \mid U \in \mathcal{U}\right\}$. Also, define

$$
\bigvee_{i=0}^{n-1} f^{-i}(\mathcal{U})=\mathcal{U} \vee f^{-1}(\mathcal{U}) \vee \ldots \vee f^{-n+1}(\mathcal{U}) \text {, where } f^{0}=\text { id }
$$

and

$$
\operatorname{Ent}(f, \mathcal{U})=\lim _{n \rightarrow \infty}(1 / n) \log N\left(\bigvee_{i=0}^{n-1} f^{-i}(\mathcal{U})\right)
$$

Then the topological entropy of $f$ is defined as

$$
\operatorname{Ent}(f)=\sup \{\operatorname{Ent}(f, \mathcal{U}) \mid \mathcal{U} \text { is an open cover of } X\} \text {. }
$$

\section{INDEPENDENCE SETS}

In this section, we look at the relationship between the entropy of a function and independence sets for a collection of disjoint open sets. For more on independence sets, see 3 .

Let $G=\{g(i)\}_{i=1}^{\infty}$ be an increasing sequence of positive integers. $G$ has positive density if $\lim _{n \rightarrow \infty} \frac{|G \cap\{1,2, \ldots, n\}|}{n}$ exists and has positive value.

Proposition 1. If $G$ has positive density, then there exists $r>1$ such that $g(n)<$ $r n$ for all $n$. Conversely, if $g(n)<r n$ for all $n$, then a subsequence of $G$ has positive density.

Proof. Suppose that $\lim _{n \rightarrow \infty} \frac{|G \cap\{1,2, \ldots, n\}|}{n}=\alpha$. Then there exists an integer $N$ such that $|G \cap\{1,2, \ldots, n\}|>\frac{\alpha}{2} n$ for all $n \geq N$. Thus $g(\lfloor n \alpha / 2\rfloor) \leq n$ and it follows that $g(n) \leq\lceil 2 / \alpha\rceil n$. Letting $r>\lceil 2 / \alpha\rceil n$, the first part of the proposition follows.

Next suppose there exists $r>0$ such that $g(n)<r n$ for all $n$. Thus,

and hence

$$
\frac{|G \cap\{1,2, \ldots, r n\}|}{r n} \geq \frac{n}{r n}=\frac{1}{r}
$$

$$
1 \geq \limsup _{n \rightarrow \infty} \frac{|G \cap\{1,2, \ldots, n\}|}{n} \geq \frac{1}{r}>0 .
$$

Therefore, there exists a subsequence of $G$ that has positive density.

Proposition 2. Suppose that $G=\{g(i)\}_{i=1}^{\infty}$ is an increasing sequence with positive density and such that $g(i+1)-g(i)>k$ for each $i$ and some positive integer $k$. Suppose further that $G^{\prime}=\left\{g^{\prime}(i)\right\}_{i=1}^{\infty}$ is defined by $g^{\prime}(2 i)=g(i)$ and $g^{\prime}(2 i-1)=$ $g(i)-k$. Then $G^{\prime}$ has positive density.

Proof. This follows from the fact that

$$
\begin{aligned}
\lim _{n \rightarrow \infty} \frac{\left|G^{\prime} \cap\{1,2, \ldots, n\}\right|}{n} & =2 \lim _{n \rightarrow \infty} \frac{\left|G^{\prime} \cap\{1,2, \ldots, n\}\right|}{2 n} \\
& =2 \lim _{n \rightarrow \infty} \frac{|G \cap\{1,2, \ldots, n\}|}{n} .
\end{aligned}
$$

Let $f: X \longrightarrow X$ be a map of a continuum $X$ and $\left\{A_{1}, \ldots, A_{k}\right\}$ be subsets of $X$. We say that $G$ is an independence set for $\left\{A_{1}, \ldots, A_{k}\right\}$ under $f$ if for every finite sequence $\left\{Y_{i}\right\}_{i=1}^{n}$, where $Y_{i} \in\left\{A_{1}, \ldots, A_{k}\right\}$, we have

$$
\bigcap_{i=1}^{n} f^{-g(i)}\left(Y_{i}\right) \neq \emptyset
$$


An ordered pair $(x, y) \in X \times X$ is an $I E$-pair if for every product neighborhood $A \times B$ of $(x, y)$ the pair $\{A, B\}$ has an independence set of positive density. Let $\operatorname{IE}_{2}(X, f)$ denote the set of IE-pairs generated by $f$. Let $\Delta_{2}(X)=\{(x, x) \in X \times X \mid x \in X\}$.

The following theorem is Proposition 3.9 (part (2)) in [3]:

Theorem 3. $I E_{2}(X, f)-\Delta_{2}(X) \neq \emptyset$ if and only if $\operatorname{Ent}(f)>0$.

Corollary 4. Suppose that $f: X \longrightarrow X$ is a map such that $\operatorname{Ent}(f)>0$. Then there exist open sets $A, B$ with $\bar{A} \cap \bar{B}=\emptyset$ such that $\{A, B\}$ has an independence set of positive density.

Proof. By Theorem 3 , there exists $(x, y) \in I E_{2}(X, f) \backslash \Delta_{2}(X)$. Then $x \neq y$. Since $(x, y)$ is an IE-pair, there exist open sets with disjoint closures about $x$ and $y$, such that the pair $\{A, B\}$ has an independence set of positive density.

The next theorem is Lemma 3.8 in [3]:

Theorem 5. Suppose that $\left\{A_{1}, \ldots, A_{k}\right\}$ has an independence set of positive density and $A_{1}=\widehat{A}_{1} \cup \widehat{A}_{2}$. Then at least one of the following is true:

(1) $\left\{\widehat{A}_{1}, A_{2}, \ldots, A_{k}\right\}$ has an independence set of positive density.

(2) $\left\{\widehat{A}_{2}, A_{2} \ldots, A_{k}\right\}$ has an independence set of positive density.

Corollary 6. Suppose that $\left\{A_{1}, \ldots, A_{k}\right\}$ has an independence set of positive density and that $\mathcal{A}_{1}, \ldots, \mathcal{A}_{k}$ are finite collections of open sets such that $A_{i} \subset \bigcup_{A^{\prime} \in \mathcal{A}_{i}} A^{\prime}$ for each $i \in\{1, \ldots, k\}$. Then for each $i$ there exists $\widehat{A}_{i} \in \mathcal{A}_{i}$ such that $\left\{\widehat{A}_{1}, \ldots, \widehat{A}_{k}\right\}$ has an independence set of positive density.

Proof. This follows from repeated applications of Theorem 5 .

Corollary 7. Let $f: X \longrightarrow X$ be a map of a chainable continuum with positive entropy. Then there exists a chain cover $\mathcal{C}, C_{i}, C_{j} \in \mathcal{C}$ with $\overline{C_{i}} \cap \overline{C_{j}}=\emptyset$ and an increasing sequence $G^{\prime}$ such that $G^{\prime}$ is an independence set for $\left\{C_{i}, C_{j}\right\}$ with positive density.

Proof. By Corollary 4 , there exist open sets $A, B$ with $\bar{A} \cap \bar{B} \neq \emptyset$ such that $\{A, B\}$ has an independence set $G$ of positive density. Choose $\delta>0$ such that

$$
\delta<\frac{1}{3} \mathrm{~d}(A, B) .
$$

Let $\mathcal{C}$ be a chain cover of $X$ with mesh less than $\delta$. Then let $\mathcal{C}(A)=\{C \in$ $\mathcal{C} \mid A \cap C \neq \emptyset\}$ and $\mathcal{C}(B)=\{C \in \mathcal{C} \mid B \cap C \neq \emptyset\}$. By Corollary 6 there exists an increasing subsequence $G^{\prime}$ of $G, C_{i} \in \mathcal{C}(A)$ and $C_{j} \in \mathcal{C}(B)$ such that $G^{\prime}$ is an independence set for $\left\{C_{i}, C_{j}\right\}$ with positive density. Since $\mathrm{d}(A, B)>3 \delta$ it follows that $\overline{C_{i}} \cap \overline{C_{j}}=\emptyset$.

Positive integers $k_{1}<k_{2}$ are called a recurrent action pair for $\{A, B\}$ under $f$ if for every 4-tuple $\left(Y_{1}, Y_{2}, Y_{3}, Y_{4}\right)$ of $\Pi_{i=1}^{4}\{A, B\}$ we have

$$
Y_{1} \cap f^{-k_{1}}\left(Y_{2}\right) \cap f^{-k_{2}}\left(Y_{3}\right) \cap f^{-k_{1}-k_{2}}\left(Y_{4}\right) \neq \emptyset .
$$

If $k_{1}, k_{2}$ are a recurrent action pair for $\{A, B\}$ under $f$, then let

$$
R_{k_{1}, k_{2}}^{f}(A, B)=\left\{Y_{1} \cap f^{-k_{1}}\left(Y_{2}\right) \cap f^{-k_{2}}\left(Y_{3}\right) \cap f^{-k_{1}-k_{2}}\left(Y_{4}\right) \mid Y_{i} \in\{A, B\}\right\} .
$$


We say that the sequence $G$ has a recurrent pattern $\left\{k_{1}, k_{2}\right\}$ if $k_{1}<k_{2}$ and

$$
\begin{gathered}
g(2 i)-g(2 i-1)=k_{1}, \\
g(4 i)-g(4 i-2)=k_{2}
\end{gathered}
$$

for all $i$.

Lemma 8. Suppose that $G=\{g(i)\}_{i=1}^{\infty}$ is an increasing sequence of positive integers that has positive density. Then there exists a subsequence $G^{\prime}=\left\{g^{\prime}(i)\right\}_{i=1}^{\infty}$ of $G$ and a positive integer $k$ such that $G^{\prime}$ has positive density and $g^{\prime}(2 i)-g^{\prime}(2 i-1)=k$ for all $i$.

Proof. Since $G$ has positive density, by Proposition 1 there exists an integer $r>1$ such that $g(i) \leq r i$ for all $i$. Let $I_{m}, J_{m}$ be a partition of $\{1, \ldots, m\}$ such that

$$
I_{m}=\{i \leq m \mid g(i+1)-g(i) \leq r\}
$$

and

$$
J_{m}=\{i \leq m \mid g(i+1)-g(i) \geq r+1\} .
$$

Claim 8.1. $\left|I_{(r+1) p}\right| \geq p$.

Suppose on the contrary that $\left|I_{(r+1) p}\right| \leq p-1$. Then

$$
\left|J_{(r+1) p}\right|=(r+1) p-\left|I_{(r+1) p}\right| \geq(r+1) p-p+1
$$

and $\left|J_{(r+1) p}\right| \leq(r+1) p$. So it follows that

$$
\begin{aligned}
g((r+1) p+1) & >g((r+1) p+1)-g(1) \\
& =\sum_{i=1}^{(r+1) p} g(i+1)-g(i) \\
& \geq \sum_{i \in J_{(r+1) p}} g(i+1)-g(i) \\
& \geq(r+1)[(r+1) p-p+1] \\
& =r(r+1) p+r+1 \\
& >r(r+1) p+r \\
& =r[(r+1) p+1] .
\end{aligned}
$$

However, the above inequality violates the hypothesis that $g(i) \leq r i$. Thus the claim is true.

For $k \in\{1, \ldots, r\}$ define

$$
I_{m}^{k}=\{i \leq m \mid g(i+1)-g(i)=k\} .
$$

Then

$$
\left|I_{m}\right|=\left|I_{m}^{1}\right|+\ldots+\left|I_{m}^{r}\right|
$$

Let $m=(r+1) p$. Then $p=m /(r+1)$. Therefore, it follows from the claim that for each $m$ there is a $k_{m} \in\{1, \ldots, r\}$ such that

$$
\left|I_{m}^{k_{m}}\right| \geq \frac{\left|I_{m}\right|}{r} \geq \frac{m}{r(r+1)} .
$$

Furthermore, there is an increasing sequence $\{m(j)\}_{j=1}^{\infty}$ such that each $k_{m(j)}$ has the same value $k$. Note that if $m \leq n$, then $I_{m}^{k} \subset I_{n}^{k}$. Let $I^{k}=\bigcup_{m=1}^{\infty} I_{m}^{k}=\left\{n_{i}\right\}_{i=1}^{\infty}$, 
where $n_{i+1}>n_{i}$. Now for each $i$, define $g_{1}(2 i)=g\left(n_{i}+1\right)$ and $g_{1}(2 i-1)=g\left(n_{i}\right)$. Then $g_{1}(2 i)-g_{1}(2 i-1)=k$. Notice that $n_{i}$ is the maximum of $I_{n_{i}}^{k}$ and that

$$
i=\left|I_{n_{i}}^{k}\right| \geq \frac{n_{i}}{r(r+1)} .
$$

So

$$
g_{1}(2 i) \leq g_{1}(2(2 i)-1)=g\left(n_{2 i}\right) \leq r n_{2 i} \leq r^{2}(r+1) 2 i .
$$

Thus, by Proposition 1, there exists an increasing sequence $\left\{i_{m}\right\}_{m=1}^{\infty}$ such that $\left\{g_{1}\left(2 i_{m}\right)\right\}_{m=1}^{\infty}$ has positive density. Let $g^{\prime}(2 m)=g_{1}\left(2 i_{m}\right)$ and $g^{\prime}(2 m-1)=$ $g_{1}\left(2 i_{m}-1\right)$. Then $G^{\prime}$ has positive density by Proposition 2 and

$$
g^{\prime}(2 m)-g^{\prime}(2 m-1)=g_{1}\left(2 i_{m}\right)-g_{1}\left(2 i_{m}-1\right)=k
$$

for each $m$.

Theorem 9. If $G$ has positive density, then there exists a subsequence $G^{\prime}$ and integers $k_{1}<k_{2}$ such that $G^{\prime}$ has positive density and recurrent pattern $\left\{k_{1}, k_{2}\right\}$.

Proof. By Lemma 8 there exists a positive integer $k_{1}$ and a subsequence $G_{1}=$ $\left\{g_{1}(i)\right\}_{i=1}^{\infty}$ of $G$ with positive density such that $g_{1}(2 i)-g_{1}(2 i-1)=k_{1}$ for all $i$. Also by Lemma 8 , there is a positive integer $k_{2}$ and an increasing sequence $\left\{i_{n}\right\}_{n=1}^{\infty}$ such that $\left\{g_{1}\left(2 i_{n}\right)\right\}_{n=1}^{\infty}$ has positive density and $g_{1}\left(2 i_{2 n}\right)-g_{1}\left(2 i_{2 n-1}\right)=k_{2}$ for all $n$. Since

$$
g_{1}\left(2 i_{2 n-1}\right)<g_{1}\left(2 i_{2 n}-1\right)<g_{1}\left(2 i_{2 n}\right),
$$

it follows that $k_{2}>k_{1}$. For each $n$ let $g^{\prime}(4 n-3)=g_{1}\left(2 i_{2 n-1}-1\right), g^{\prime}(4 n-2)=$ $g_{1}\left(2 i_{2 n-1}\right), g^{\prime}(4 n-1)=g_{1}\left(2 i_{2 n}-1\right)$ and $g^{\prime}(4 n)=g_{1}\left(2 i_{2 n}\right)$. Then $G^{\prime}=\left\{g^{\prime}(i)\right\}_{i=1}^{\infty}$ has positive density and recurrent pattern $\left\{k_{1}, k_{2}\right\}$.

Corollary 10. Suppose $\{A, B\}$ has an independence set $G$ of positive density. Then there exist positive integers $k_{1}<k_{2}$ and an independence set $G_{1}$ of positive density for $R_{k_{1}, k_{2}}^{f}(A, B)$.

Proof. By Theorem 9 there exist integers $k_{1}<k_{2}$ and a subsequence $G^{\prime}=\left\{g^{\prime}(i)\right\}_{i=1}^{\infty}$ of positive density with recurrent pattern $\left\{k_{1}, k_{2}\right\}$. Notice that if $\left\{\widehat{Y}_{i}\right\}_{i=1}^{n}$ is a sequence of terms from $R_{k_{1}, k_{2}}^{f}(A, B)$, then there exists a sequence of terms, $\left\{Y_{i}\right\}_{i=1}^{4 n}$, from $\{A, B\}$ such that

$$
\bigcap_{i=1}^{n} f^{-g^{\prime}(4 i-3)}\left(\widehat{Y}_{i}\right)=\bigcap_{i=1}^{4 n} f^{-g^{\prime}(i)}\left(Y_{i}\right) \neq \emptyset .
$$

Let $g_{1}(i)=g^{\prime}(4 i-3)$. Then $G_{1}=\left\{g_{1}(i)\right\}_{i=1}^{\infty}$ is an independence set of positive density for $R_{k_{1}, k_{2}}^{f}(A, B)$.

Theorem 11. Suppose that $f: X \longrightarrow X$ is a map and that $A, B$ are open sets with an independence set $G$ of positive density and such that $\bar{A} \cap \bar{B}=\emptyset$. Then there exist integers $k_{1}<k_{2}$ and $\delta>0$ such that if $\mathcal{U}$ is a finite open cover of $X$ with mesh $(\mathcal{U})<\delta$, then there exist disjoint $V, U_{1}, \ldots, U_{9} \in \mathcal{U}$ with the following properties:

(1) $\bar{V} \cap \bar{U}_{i}=\emptyset$ for each $i \in\{1, \ldots, 9\}$,

(2) $\bar{U}_{i} \cap \bar{U}_{j}=\emptyset$ for $i \neq j$,

(3) $\left\{V, U_{1}, \ldots, U_{9}\right\}$ has an independence set of positive density,

(4) each element of $\left\{V, U_{1}, \ldots, U_{9}\right\}$ intersects a unique element of $R_{k_{1}, k_{2}}^{f}(A, B)$. 
Proof. It follows from Corollary 10 that there exist integers $k_{1}<k_{2}$ and an increasing sequence $G^{\prime}$ that is an independence set for $R_{k_{1}, k_{2}}^{f}(A, B)$ with positive density. Let $\left\{Y_{0}, \ldots, Y_{15}\right\}$ be the distinct elements of $R_{k_{1}, k_{2}}^{f}(A, B)$. Since $\bar{A} \cap \bar{B}=\emptyset$, it follows that $\overline{Y_{i}} \cap \overline{Y_{j}}=\emptyset$ for all $i \neq j$. So choose $\delta>0$ and such that

$$
\delta<\frac{1}{3} \min \left\{\mathrm{d}\left(Y_{i}, Y_{j}\right) \mid i \neq j\right\} .
$$

Let $\mathcal{U}$ be a chain cover of $X$ with mesh less than $\delta$. Let $\mathcal{C}\left(Y_{i}\right)=\left\{U \in \mathcal{U} \mid Y_{i} \cap U \neq \emptyset\right\}$. Then by Corollary 6 there exists an increasing subsequence $G_{1}$ of $G^{\prime}, V \in \mathcal{C}\left(Y_{0}\right)$ and $U_{i} \in \mathcal{C}\left(Y_{i}\right)$ for $i \in\{1, \ldots, 9\}$ such that $G_{1}$ is an independence set for $\left\{V, U_{1}, \ldots, U_{9}\right\}$ with positive density. Each element of $\left\{V, U_{1}, \ldots, U_{9}\right\}$ clearly intersects a unique element of $R_{k_{1}, k_{2}}^{f}(A, B)$ and since $\mathrm{d}\left(Y_{i}, Y_{j}\right) \geq 3 \delta$ it follows that $\bar{V} \cap \bar{U}_{i}=\emptyset$ for each $i \in\{1, \ldots, 9\}$ and $\bar{U}_{i} \cap \bar{U}_{j}=\emptyset$ for $i \neq j$.

\section{MAIN RESUlt}

This section shows the relationship between entropy and crookedness. In order for a chainable continuum to be indecomposable it must consist of nested crooked chains. Let $\mathcal{C}_{0}$ and $\mathcal{C}_{1}=\left[C_{1}^{1}, C_{2}^{1}, \ldots, C_{n}^{1}\right]$ be chains such that $\mathcal{C}_{1}$ refines $\mathcal{C}_{0}$. If $A$ and $B$ are disjoint elements of $\mathcal{C}_{0}$, then $\mathcal{C}_{1}$ is crooked between $A$ and $B$ if there exist $i<j<k$ such that either

(1) $C_{i}^{1} \cap A \neq \emptyset, C_{k}^{1} \cap A \neq \emptyset$, and $C_{j}^{1} \cap B \neq \emptyset$ or

(2) $C_{i}^{1} \cap B \neq \emptyset, C_{k}^{1} \cap B \neq \emptyset$, and $C_{j}^{1} \cap A \neq \emptyset$.

Next define

$$
\mathcal{C}^{*}=\bigcup_{C \in \mathcal{C}} C
$$

The next theorem gives the connection between crookedness and indecomposablity and can be found in [1] in a slightly different form.

Theorem 12. Let $\mathcal{C}_{0}$ be a chain cover of the continuum $X, A_{0}, B_{0} \in \mathcal{C}_{0}$ such that $A_{0} \cap B_{0}=\emptyset$ and $\epsilon=\frac{1}{3} d\left(A_{0}, B_{0}\right)$. Suppose that for each $i$ there exists a chain cover $\mathcal{C}_{i}$ and $A_{i}, B_{i} \in \mathcal{C}_{i}$ such that

(1) $\operatorname{mesh}\left(\mathcal{C}_{i}\right)<\epsilon / 3^{i}$,

(2) $\mathcal{C}_{i}$ closure refines $\mathcal{C}_{i-1}$,

(3) the subchain of $\mathcal{C}_{i}$ between $A_{i}$ and $B_{i}$ is crooked between $A_{i-1}$ and $B_{i-1}$. Then $X=\bigcap_{i \rightarrow \infty} \mathcal{C}_{i}^{*}$ contains a nondegenerate indecomposable subcontinuum.

Let $W=\left\langle U_{i}\right\rangle_{i=1}^{n}$ be a finite sequence of symbols. Define $\pi_{i}(W)=U_{i}$. The next two technical lemmas will aid in proving the main result.

Lemma 13. Let $\mathcal{W}_{4}=\left\{\left\langle U_{i}\right\rangle_{i=1}^{4} \mid U_{i} \in\{A, B\}\right\}$ and suppose that $W_{1}, \ldots, W_{6}$ are six distinct elements of $\mathcal{W}_{4}$. Then there exists $i \in\{1, \ldots, 4\}$ and $1<j<k$ such that $\pi_{i}\left(W_{1}\right)=\pi_{i}\left(W_{k}\right)$ and $\pi_{i}\left(W_{1}\right) \neq \pi_{i}\left(W_{j}\right)$.

Proof. Since the sequences are comprised of only two symbols, it suffices to show that there exists an $i^{\prime}$ and $1<j<k$ such that $\pi_{i^{\prime}}\left(W_{1}\right) \neq \pi_{i^{\prime}}\left(W_{j}\right)$ and $\pi_{i^{\prime}}\left(W_{j}\right) \neq$ $\pi_{i^{\prime}}\left(W_{k}\right)$. Suppose on the contrary that for each $i \in\{1, \ldots, 4\}$ there is at most one integer $n_{i} \in\{1, \ldots, 6\}$ such $\pi_{i}\left(W_{n_{i}}\right) \neq \pi_{i}\left(W_{n_{i}+1}\right)$. Let

$$
\Psi(i)= \begin{cases}n_{i} & \text { if } \pi_{i}\left(W_{n_{i}}\right) \neq \pi_{i}\left(W_{n_{i}+1}\right), \\ 0 & \text { if } \pi_{i}\left(W_{j}\right)=\pi_{i}\left(W_{k}\right) \text { for every } j, k \in\{1, \ldots, 6\} .\end{cases}
$$


Let $\left\{i_{k}\right\}_{k=1}^{4}=\{1, \ldots, 4\}$ be a finite sequence such that $\Psi\left(i_{k}\right) \leq \Psi\left(i_{k+1}\right)$ for all $k$. Notice that $0 \leq \Psi\left(i_{k}\right) \leq 5$. Then by the pigeon-hole principle one of the following must be true:

(1) $\Psi\left(i_{1}\right) \geq 2$,

(2) there must be a $k$ such that $\Psi\left(i_{k+1}\right)-\Psi\left(i_{k}\right) \geq 2$,

(3) $\Psi\left(i_{4}\right) \leq 4$.

However, that implies that one of the following, respectively, is true:

(1) $W_{1}=W_{2}$,

(2) $W_{\Psi\left(i_{k+1}\right)-1}=W_{\Psi\left(i_{k+1}\right)}$,

(3) $W_{5}=W_{6}$.

This is a contradiction in each case.

Lemma 14. Let $h: X \longrightarrow X$ be a homeomorphism, $A, B$ be open sets such that $\bar{A} \cap \bar{B}=\emptyset$, and $\mathcal{U}=\left[U_{1}, \ldots, U_{p_{1}}, \ldots, U_{p_{2}}, \ldots, U_{p_{6}}, \ldots, U_{m}\right]$ be a chain cover of $X$ such that $\left\{U_{p_{i}}\right\}_{i=1}^{6}$ all intersect unique elements of $R_{k_{1}, k_{2}}^{h}(A, B)$. Then there exist $1<j<k \leq 6$ and $n \in\left\{0, k_{1}, k_{2}, k_{1}+k_{2}\right\}$ such that either

(1) $h^{n}\left(U_{p_{1}}\right) \cap B \neq \emptyset, h^{n}\left(U_{p_{k}}\right) \cap B \neq \emptyset$, and $h^{n}\left(U_{p_{j}}\right) \cap A \neq \emptyset$ or

(2) $h^{n}\left(U_{p_{1}}\right) \cap A \neq \emptyset, h^{n}\left(U_{p_{k}}\right) \cap A \neq \emptyset$, and $h^{n}\left(U_{p_{j}}\right) \cap B \neq \emptyset$.

Proof. Let $W_{\alpha}=\left\langle Y_{1}^{\alpha}, Y_{2}^{\alpha}, Y_{3}^{\alpha}, Y_{4}^{\alpha}\right\rangle$ be the sequence associated with the unique element

$Y_{1}^{\alpha} \cap h^{-k_{1}}\left(Y_{2}^{\alpha}\right) \cap h^{-k_{2}}\left(Y_{3}^{\alpha}\right) \cap h^{-k_{1}-k_{2}}\left(Y_{4}^{\alpha}\right)=\left(Y_{1}^{\alpha} \cap h^{-k_{1}}\left(Y_{2}^{\alpha}\right)\right) \cap h^{-k_{2}}\left(Y_{3}^{\alpha} \cap h^{-k_{1}}\left(Y_{4}^{\alpha}\right)\right)$ of $R_{k_{1}, k_{2}}^{h}(A, B)$ that intersects $U_{p_{\alpha}}$, where $Y_{i}^{\alpha} \in\{A, B\}$. Then by Lemma 13, there exists $1<j<k$ and $i^{\prime} \in\{1, \ldots, 4\}$ such that $Y_{i^{\prime}}^{1}=Y_{i^{\prime}}^{k}$ and $Y_{i^{\prime}}^{1} \neq Y_{i^{\prime}}^{j}$.

First, suppose that $Y_{i^{\prime}}^{1}=Y_{i^{\prime}}^{k}=B$ and $Y_{i^{\prime}}^{j}=A$. Then using the value of $n_{i^{\prime}}$ defined by $n_{1}=0, n_{2}=k_{1}, n_{3}=k_{2}$ and $n_{4}=k_{1}+k_{2}$, it follows that

$$
U_{p_{1}} \cap h^{-n_{i^{\prime}}}(B) \neq \emptyset, U_{p_{k}} \cap h^{-n_{i^{\prime}}}(B) \neq \emptyset \text { and } U_{p_{j}} \cap h^{-n_{i^{\prime}}}(A) \neq \emptyset,
$$

so (1) is true. On the other hand, if $Y_{i^{\prime}}^{1}=Y_{i^{\prime}}^{k}=A$ and $Y_{i^{\prime}}^{j}=B$, then (2) follows in a similar manner.

The next lemma shows the relationship between entropy and crookedness.

Lemma 15. Suppose that $h: X \longrightarrow X$ is a homeomorphism of a chainable continuum and that $A, B$ are disjoint elements of a proper chain cover $\mathcal{C}$ such that $\{A, B\}$ have an independence set of positive density. Then for every $\epsilon>0$, there exists a chain cover $\mathcal{U}$ and elements $U, V \in \mathcal{U}$ with the following properties:

(1) $\operatorname{mesh}(\mathcal{U})<\epsilon$.

(2) $\{U, V\}$ have an independence set of positive density.

(3) The subchain from $U$ to $V$ is crooked between $A$ and $B$.

Proof. Let $\epsilon>0$. Then by Theorem 9, there exist an increasing sequence of positive integers $G=\{g(i)\}_{i=1}^{\infty}$ and positive integers $k_{1}, k_{2}$ such that $G$ is an independence set of positive density for $R_{k_{1}, k_{2}}^{h}(A, B)$. By uniform continuity, there exists a $\delta>0$ such that if $d(x, y)<\delta$, then $d\left(h^{n}(x), h^{n}(y)\right)<\epsilon$ for all $-k_{1}-k_{2} \leq n \leq k_{1}+k_{2}$. Let $\mathcal{U}$ be a chain cover of $X$ with mesh less than $\delta$. Then by Theorem 11, we can assume that there exist distinct elements $V, U_{1}, \ldots, U_{9}$ of $\mathcal{U}$ such that each element intersects a unique element of $R_{k_{1}, k_{2}}^{h}(A, B)$ and $\left\{V, U_{1}, \ldots, U_{9}\right\}$ has an independence set of positive density. 
In the ordering of chain $\mathcal{U}$, at least 5 elements of $\left\{U_{1}, \ldots, U_{9}\right\}$ either follow $V$ or precede $V$. Since each element of $R_{k_{1}, k_{2}}^{h}(A, B)$ is of the form $Y_{1} \cap h^{-k_{1}}\left(Y_{2}\right) \cap$ $h^{-k_{2}}\left(Y_{3}\right) \cap h^{-k_{1}-k_{2}}\left(Y_{4}\right)$, where $Y_{i} \in\{A, B\}$, it follows from Lemma 14 that there exists $j \in\left\{0, k_{0}, k_{1}, k_{0}+k_{1}\right\}$ and $U_{a}, U_{b}$ such that

1) $U_{a}$ is between $V$ and $U_{b}$ in the ordering of $\mathcal{U}$ and either

2) $h^{j}(V) \cap B \neq \emptyset, h^{j}\left(U_{b}\right) \cap B \neq \emptyset$, and $h^{j}\left(U_{a}\right) \cap A \neq \emptyset$ or

$\left.2^{\prime}\right) h^{j}(V) \cap A \neq \emptyset, h^{j}\left(U_{b}\right) \cap A \neq \emptyset$, and $h^{j}\left(U_{a}\right) \cap B \neq \emptyset$.

Hence, the lemma follows.

The next theorem is the main result of this paper.

Theorem 16. Suppose that $X$ is a chainable continuum and $h: X \longrightarrow X$ is a homeomorphism such that $\operatorname{Ent}(h)>0$. Then $X$ must contain an indecomposable subcontinuum.

Proof. By Corollary 7 there exists a chain cover $\mathcal{C}_{0}$ of $X$ and $A, B \in \mathcal{C}_{0}$ such that $\bar{A} \cap \bar{B}=\emptyset$ and $\{A, B\}$ has an independence set of positive density. Let $0<\gamma<\frac{1}{3} \mathrm{~d}(A, B)$.

Continuing inductively suppose that a chain cover $\mathcal{C}_{i}$ has been found with the following properties:

(1) $\operatorname{mesh}\left(\mathcal{C}_{i}\right)<\gamma / 3^{i}$

(2) there exist $A_{i}, B_{i} \in \mathcal{C}_{i}$ such that $\overline{A_{i}} \cap \overline{B_{i}}=\emptyset$ and $\left\{A_{i}, B_{i}\right\}$ has an independence set of positive density.

Then by Lemma 15, there exists a chain-cover $\mathcal{C}_{i+1}$ such that

(1) $\mathcal{C}_{i+1}$ closure refines $\mathcal{C}_{i}$;

(2) $\operatorname{mesh}\left(\mathcal{C}_{i+1}\right)<\gamma / 3^{i+1}$;

(3) there exist $A_{i+1}, B_{i+1} \in \mathcal{C}_{i+1}$ such that $\overline{A_{i+1}} \cap \overline{B_{i+1}}=\emptyset$ and $\left\{A_{i+1}, B_{i+1}\right\}$ has an independence set of positive density;

(4) the subchain of $\mathcal{C}_{i+1}$ between $A_{i+1}$ and $B_{i+1}$ is crooked between $A_{i}$ and $B_{i}$. Since $X=\bigcap_{i \rightarrow \infty} \mathcal{C}_{i}^{*}$, it follows from Theorem 12 that $X$ contains a nondegenerate indecomposable subcontinuum.

A function $f$ is open if $f(U)$ is open for each open set $U$ in the domain of $f$. A function $f$ is monotone if $f^{-1}(H)$ is connected for every connected $H$ in the range of $f$. Homeomorphisms are monotone and open. So the following are natural questions:

Question 1. If $f: X \longrightarrow X$ is a monotone open continuous function of a chainable continuum such that $\operatorname{Ent}(f)>0$, then must $X$ contain a nondegenerate indecomposable subcontinuum?

If so, then consider the next question.

Question 2. If $f: X \longrightarrow X$ is a monotone continuous function of a chainable continuum such that $\operatorname{Ent}(f)>0$, then must $X$ contain a nondegenerate indecomposable subcontinuum?

However, it is well known that the tent map $f:[0,1] \longrightarrow[0,1]$ defined by

$$
f(x)= \begin{cases}2 x & \text { if } x \in[0,1 / 2], \\ 2-2 x & \text { if } x \in(1 / 2,1]\end{cases}
$$


is an open continuous function such that $\operatorname{Ent}(f)=\log (2)$. Also, the cone over the Cantor set (also known as the Cantor Fan) admits a positive entropy homeomorphism. The cone over the Cantor set is tree-like but not chainable and does not contain a nondegenerate indecomposable subcontinuum.

\section{REFERENCES}

1. Lida K. Barrett, The structure of decomposable snakelike continua, Duke Math. J. 28 (1961), 515-522. MR0133114 (24:A2948)

2. Marcy Barge and Morton Brown, Problems in dynamics on continua. Contemporary Mathematics 117 (A.M.S., Providence, RI, 1991). MR.1112814(92i:54032)

3. David Kerr and Hanfeng Li, Independence in topological and $C^{*}$-dynamics, Mathematische Annalen, 338, August 2007, 869-926. MR2317754(2009a:46126)

4. G. T. Seidler, The topological entropy of homeomorphisms on one-dimensional continua, Proc. Amer. Math. Soc. 4 (1990), 1025-1030. MR946627 (90g:54012)

5. X. Ye, Topological entropy of the induced maps of the inverse limits with bonding maps, Topology Appl. 67 (1995), no. 2, 113-118. MR1362077 (97j:54046)

6. X. Ye, The topological entropy of homeomorphisms of hereditarily decomposable chainable continua, Dynamical systems and chaos, Vol. 1 (Hachioji, 1994), 290-293. MR1479947 (98f:54029)

Department of Mathematics and Computer Science, Rhodes College, Memphis, TenNESSEE 38112

E-mail address: mouronc@rhodes.edu 